\title{
Review of: "Prevalence of nutritional risk and malnutrition during and after hospitalization for COVID-19 infection: Preliminary results of a single- centre experience"
}

\author{
Sabina Mikkelsen
}

Potential competing interests: The author(s) declared that no potential competing interests exist.

This is a single-centre experience, which examined the prevalence of nutritional risk and malnutrition during and after hospitalization for COVID-19 infection. They measured the patients at three timepoints: upon admission, at discharge and at three months follow-up. They compared the patients admitted to an Intensive Care Unit (ICU), Intermediate Care Unit (IMCU) or standard care wards. This study is very interesting with some interesting results. In this study 142 patients were recruited, which can affect how representative the results are and how strong the conclusion is, as the authors performed analyzes where the patients were classified into smaller groups, so the groups may be small.

There are some concerns with this study which include the following:

1. The conclusion is that admission to an ICU/IMCU seem to have a major impact on nutritional status, but there is no table or description of how many were admitted to ICU, IMCU and standard care wards respectively. If there only were two admitted at the standard care units, this may explain the results, but we don't know.

2. There are no demographic table about the participants, so we do not know, how many have had an UWL (n). This make it difficult to perspective the results to other countries.

3. In the conclusion the authors state that patients how lose more than $10 \%$ of their habitual weight during hospital stay were at higher nutritional risk. This statement was not mention in the results or discussion section, which can be an issue, as the statement should be discussed.

4. The authors should be consistent when they present data (one or two decimal) and use the same term e.g. they use different word for a standard care ward, which I think they should, as it can be confusing for the reader.

5. The authors could advantageously discuss their strength and limitations, so the readers can see, that they have made some considerations about it.

Finally, I think the study have some interesting results, but there are some concerns with this study. 